\title{
Normal FeNO: What Do We Know?
}

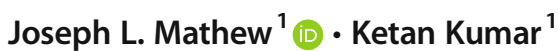

Received: 12 May 2021 / Accepted: 19 May 2021 / Published online: 7 June 2021

(C) Dr. K C Chaudhuri Foundation 2021

Bronchial asthma is the most common cause of pediatric hospital visits and school absenteeism, globally [1]. In India, the prevalence of asthma is estimated as 10\%-15\% among children between the ages of 5 and 11 y [2]. Traditionally, childhood asthma is diagnosed clinically, by the characteristic pattern of episodic, reversible respiratory symptoms, including wheezing, cough, shortness of breath, or chest tightness. Objective measurements of lung function are challenged by variability in symptoms, technical difficulties, limited access to facilities, and relative ease of treatment using inhaled corticosteroids. However, recognition of distinct asthma endotypes, makes it potentially feasible to use a personalized medicine approach for treatment. This requires identification of the inflammatory pathway in individual patients, and targeted therapy

Against this backdrop, fractional exhaled nitric oxide ( $\mathrm{FeNO}$ ) is a biomarker that correlates with eosinophilic airway inflammation. Although FeNO may have only an adjunctive role in the diagnosis of asthma per se, it is recommended for establishing eosinophilic airway inflammation and predicting steroid responsiveness [3]. Global Initiative for Asthma (GINA) also recognizes the benefit of FeNO-guided treatment for reducing exacerbations in children with asthma [4]. However, reliable measurement of FeNO in children is hampered by issues such as measurement technique, method of analysis, instrument calibration, lack of inter-changeability of devices, etc. Similarly, interpretation of results is hampered by variations in demographic characteristics and also paucity of locally applicable normative values.

Ai et al. recently conducted a cohort study to establish normal FeNO values in 6-15-y-old school children in Chengdu city, China [5]. Their justification for the study was that normal $\mathrm{FeNO}$ values may vary by region

Joseph L. Mathew

dr.joseph.1.mathew@gmail.com

1 Department of Pediatrics, Advanced Pediatrics Centre, Post Graduate Institute of Medical Education \& Research (PGIMER),

Chandigarh 160012, India
There is good quality evidence that normal FeNO level (in children and adolescents) varies with ethnicity [6,7], necessitating a somewhat higher cutoff value in multiethnic populations [7]. However, Ai et al. enrolled children of a single nationality (Han) in only one city, limiting the validity of their results to a specific population, rather than the entire region (as they intended).

The investigators excluded children with pre-existing inflammatory (and other respiratory) conditions, based on questionnaire alone, rather than objective criteria. Further, they included only completely asymptomatic children, squandering the opportunity to assess FeNO levels in children with nonasthmatic airway inflammatory conditions, controlled asthma, etc. The latter would have provided valuable information on the difference in FeNO levels in these conditions compared to asymptomatic children, in their population (which was the objective in the first place).

The investigators recruited 600 children and excluded 59, based on their exclusion criteria. However, strangely, only 481 of the remaining 541 children were tested. The reasons and impact of this are unclear.

Ai et al. used standard methods for estimating FeNO, taking care to restrict inflammatory triggers in the testing environment and minimize confounding factors in the participants, coaching the children to perform the procedure properly. However, each child was tested only once, hence intraparticipant variation(s) cannot be assessed.

The investigators reported FeNO values of 6-11-y-old children separately from 12 to 15 -y-old children, referring to them as school-age and adolescents, respectively. The purpose of this distinction is unclear, especially as clinically insignificant differences were observed in the two groups. If the intention was to explore normative FeNO levels by age, multiple age bands should have been used for analysis.

The normal range of FeNO in asymptomatic children in this study [5] was broadly similar to data from other studies in diverse settings, although the precise cutoff to distinguish normal from abnormal varied. In this study [5], FeNO in the enrolled children ranged from 5 to $69 \mathrm{ppb}$; although the authors inexplicably reported that $28 \mathrm{ppb}$ was the upper limit of 
normal. Further, they concluded that this value can be taken as the cutoff for determining airway inflammation. This conclusion is erroneous, since the investigators missed the opportunity to determine this in their study.

Despite the various limitations, this study [5] provides food for thought and perhaps may be a starting point for larger, well-designed studies in local populations - not only to determine normal FeNO ranges, but more importantly, to assess whether therapy can be initiated, guided, modified, or tapered, based on this.

\section{Compliance of Ethical Standards}

Conflict of Interest None.

\section{References}

1. Ferrante G, La Grutta S. The burden of pediatric asthma. Front Pediatr. 2018;6:186.
2. Pal R, Dahal S, Pal S. Prevalence of bronchial asthma in Indian children. Indian J Community Med. 2009;34:310-6.

3. Dweik RA, Boggs PB, Erzurum SC, et al. American Thoracic Society Committee on Interpretation of Exhaled Nitric Oxide Levels (FENO) for Clinical Applications. An official ATS clinical practice guideline: interpretation of exhaled nitric oxide levels (FENO) for clinical applications. Am J Respir Crit Care Med. 2011;184:602-15.

4. Global Initiative for Asthma. In:Global Strategy for Asthma Management and Prevention. 2020. Available at: www.ginasthma. org. Accessed on 8 May 2021.

5. Ai T, Luo R, Zhang L, et al. The FeNO Normal value analysis of healthy children aged 6-15 years old in Chengdu. Indian J Pediatr. 2021. https://doi.org/10.1007/s12098-020-03649-6.

6. Blake TL, Chang AB, Chatfield MD, et al. Does ethnicity influence fractional exhaled nitric oxide in healthy individuals?: a systematic review. Chest. 2017;152:40-50.

7. Wang D, Wang Y, Liang H, David JE, Bray CL. Race and ethnicity have significant influence on fractional exhaled nitric oxide. Ann Allergy Asthma Immunol. 2018;120:272-7.

Publisher's Note Springer Nature remains neutral with regard to jurisdictional claims in published maps and institutional affiliations. 